ABSTRACT: Research evaluating the changes in nerve conduction with time has been limited to cross-sectional studies. We present a cohort study estimating how sensory nerve conduction study (NCS) parameters change with time when subjects are measured at two time-points. We evaluated 440 working adults by performing median and ulnar antidromic sensory NCS of both hands on two occasions, about 5.4 years (range, 4.3-7.0 years) apart. The rate of change in the NCS parameters was estimated using a mixedmodels analysis controlling for each hand, gender, age, and body mass index (BMI). After controlling for gender, age, height, and BMI, the amplitudes of the median sensory nerve action potentials (SNAP) decreased by about $2.3 \mu \mathrm{V}$, peak latencies increased by $0.11 \mathrm{~ms}$, onset latencies increased by $0.07 \mathrm{~ms}$, and conduction velocities decreased by $1.1 \mathrm{~m} / \mathrm{s}$ over 5 years. Corresponding values for the ulnar nerve were $1.75 \mu \mathrm{V}, 0.06 \mathrm{~ms}, 0.04$ $\mathrm{ms}$, and $0.71 \mathrm{~m} / \mathrm{s}$, respectively. The findings are consistent with the findings of previous cross-sectional studies. The rate of change over time was not affected by hand (dominant versus nondominant hand), gender, age, or BMI at baseline. The rate of change seen with some of the median nerve parameters was significantly greater than that with the ulnar nerve.

Muscle Nerve 29: 716-720, 2004

\title{
EFFECT OF AGING ON SENSORY NERVE CONDUCTION STUDY PARAMETERS
}

\author{
HENRY C. TONG, MD, ${ }^{1}$ ROBERT A. WERNER, MD, ${ }^{1-3}$ and ALFRED FRANZBLAU, MD ${ }^{3}$ \\ ${ }^{1}$ Department of Physical Medicine and Rehabilitation, School of Medicine, University of Michigan, \\ 325 East Eisenhower, 2nd floor, Ann Arbor, Michigan, USA \\ ${ }^{2}$ Department of Physical Medicine and Rehabilitation, Veterans Administration Hospital, \\ Ann Arbor, Michigan, USA \\ ${ }^{3}$ Department of Environmental Health Sciences, School of Public Health, University of Michigan, \\ Ann Arbor, Michigan, USA
}

Accepted 9 January 2004

It is widely accepted that nerve conduction study (NCS) parameters change with age. As a result, many electrodiagnostic laboratories have tables of normative values that are divided by age groups. The concept suggests that normal nerve functioning in a normal individual changes with aging. Many studies have attempted to quantify how NCS values change with age. Several studies have shown an association between age and decreased sensory conduction velocity in normal individuals, $, 5,7,8,11,13,15,19,23$ and an association between aging and motor conduction velocity has also been reported. ${ }^{5,6,8,9,11,13,19}$ However, all the studies used cross-sectional data gathered from subjects of different ages to infer how the NCS

\footnotetext{
Abbreviations: BMI, body mass index; ICC, intraclass correlation coefficient; NCS, nerve conduction studies; SNAP, sensory nerve action potential Key words: aging; electrodiagnostic tests; epidemiological study; nerve conduction studies; sensory nerve function

Correspondence to: H. Tong; e-mail: hct@umich.edu

(C) 2004 Wiley Periodicals, Inc.

Published online 24 March 2004 in Wiley InterScience (www.interscience. wiley.com). DOI 10.1002/mus.20026
}

parameters change with aging. A cross-sectional study design can suggest that nerve conduction parameters change with aging, but a prospective study of subjects at two different time points would be better able to determine whether NCS parameters change with time and to quantify such change.

The primary purpose of this study was to evaluate how sensory NCS parameters change with aging in a prospective cohort. The secondary purpose was to determine whether any changes with aging are affected by the initial age of the subject. Finally, we sought to determine whether other factors, such as hand dominance, gender, age, and body mass index (BMI) affect NCS parameter changes with time.

Methodology. In this study we used data collected for a previous study evaluating the prevalence of carpal tunnel syndrome. ${ }^{24}$ Nerve conduction studies were initially collected from 1000 workers from seven work sites, representing a variety of manufacturing and office environments, between June 1993 and September 1997. The protocol included bilat- 
eral electrodiagnostic testing of distal sensory responses at the wrist, self-administered questionnaires, and anthropometric measurements. Examiners were masked to data collected in other parts of the evaluation. From the initial group, 501 subjects (1002 arms) were then evaluated with the same protocol 4-7 years later. Subjects were asked about demographic data (height, weight, age, gender) and whether they had diabetes mellitus, thyroid disease, or rheumatoid arthritis. The BMI was calculated as weight (in kilograms) divided by height (in meters) squared.

Prior to the electrodiagnostic evaluation, midpalm temperatures were recorded and, if necessary, the hand was warmed to at least $32^{\circ} \mathrm{C}$. The median sensory and ulnar sensory conduction studies involved recording with standard ring recording electrodes at digits II and $\mathrm{V}$, respectively, with an interelectrode distance of $3 \mathrm{~cm}$. Antidromic stimulation was applied at the wrist $14 \mathrm{~cm}$ proximal to the active recording electrode. The latency to both the initial deflection (onset latency) and negative peak (peak latency) as well as the baseline-to-peak amplitude were recorded in accordance with the guidelines outlined by the American Association of Electrodiagnostic Medicine. ${ }^{2}$

The distal latencies and conduction velocities for the sensory responses were adjusted to a standard temperature of $32^{\circ} \mathrm{C}$. As noted by Denys, ${ }^{4}$ temperature is one of the most important factors explaining variation in nerve conduction and is the most frequent cause of false-positive or borderline findings. Even when limb temperature is controlled within an acceptable range, it still affects latency. ${ }^{15}$ For every $1{ }^{\circ} \mathrm{C}$ increase in temperature, latency decreases by 0.3 $\mathrm{ms}$. This is an approximately linear relationship between velocity and temperature from $18^{\circ} \mathrm{C}$ to $36^{\circ} \mathrm{C}$. Onset and peak latencies were adjusted for temperature differences according to the following formula, taking $32^{\circ} \mathrm{C}$ as the standard skin temperature: latency $_{\text {corrected }}=$ latency $_{\text {initial }}-0.3 \mathrm{~ms} *\left(32^{\circ} \mathrm{C}-\right.$ temperature in $\left.{ }^{\circ} \mathrm{C}\right)$. Temperature correction was not applied to the amplitude of the sensory nerve action potentials (SNAPs), because studies have shown conflicting data regarding whether skin temperature changes affect SNAP amplitude as well as the magnitude of any effect. ${ }^{14}$

Exclusion Criteria. Subjects were excluded if they reported any history of diabetes mellitus or carpal tunnel syndrome during the initial or follow-up testing. Carpal tunnel syndrome was regarded as present when a hand pain drawing was consistent with carpal tunnel syndrome and the median-to-ulnar SNAP peak latency difference was $\geq 0.8 \mathrm{~ms}$. This cut-off value is based on recently published criteria on the normal difference expected with active workers. ${ }^{15}$

Statistical Analysis. To evaluate the change in parameter values with time, a mixed-model linear regression analysis was performed using the following base model using SAS 8.0 (Cary, NG) with proc mixed. ${ }^{17}$ The NCS parameter value was the dependent variable. The independent variables were the time point at which data were gathered, gender, height, BMI, hand used, and age at initial evaluation. The NCS parameters collected were as follows: SNAP amplitude, peak latency, onset latency, and sensory conduction velocity for the median and ulnar nerves. The data of the two nerves were kept separate because they may behave differently. Subject was set as the repeated variable. Because the database included measures from each subject at two time points and the results from both hands of each subject were used, the covariate matrix structure was kept unstructured.

To determine whether the subject's age affected the rate of change, an interaction term between time and age was added to the base model. To determine whether any of the other potential predictive factors (i.e., dominant versus nondominant hand, gender, height, and BMI) were related to change over time, an interaction term between the time and the corresponding factor was added to the model. For example, to determine whether the change over time was related to gender, the interaction term "time*gender" was added to the base model.

To determine whether any change over time was nonlinear, a quadratic term for time (time*time) was entered into the model, and to determine whether the change over time was related to subject age nonlinearly, the interaction terms "time*age" and "time*age*age" were added to the model.

\section{RESULTS}

Of the 501 potential subjects, 61 subjects were excluded from the analysis because of a diagnosis of diabetes mellitus or carpal tunnel syndrome. Of the remaining 440 subjects, $312(71.1 \%)$ were female, and $391(89 \%)$ were right handed. The average age was 38.4 years at the initial screening ( $\mathrm{SD}, 9.7$; range, 19-63 years), and the average length of follow-up was 5.39 years (SD, 0.9; range, $4.3-7.0$ years). The average BMI was 27.8 (SD, 6.4; range, 17-56.6 years). The values for the initial and final median and ulnar sensory responses, adjusted only for temperature, are listed in Table 1. 
Table 1. Values for the initial and final median and ulnar sensory NCS, adjusted only for temperature.

\begin{tabular}{lcc}
\hline & $\begin{array}{c}\text { Unadjusted } \\
\text { initial value, } \\
\text { mean }(\mathrm{SD})\end{array}$ & $\begin{array}{c}\text { Unadjusted } \\
\text { final value, } \\
\text { mean (SD) }\end{array}$ \\
\hline Median sensory response & & \\
Amplitude $(\mu \mathrm{V})$ & $41.91(17.49)$ & $39.33(16.97)$ \\
Peak latency $(\mathrm{ms})$ & $3.55(0.38)$ & $3.64(0.56)$ \\
Onset latency $(\mathrm{ms})$ & $2.86(0.37)$ & $2.91(0.55)$ \\
Conduction velocity $(\mathrm{m} / \mathrm{s})$ & $49.91(6.31)$ & $49.23(6.74)$ \\
Ulnar sensory response & & \\
Amplitude $(\mu \mathrm{V})$ & $37.75(16.77)$ & $35.77(15.78)$ \\
Peak latency $(\mathrm{ms})$ & $3.46(0.31)$ & $3.50(0.50)$ \\
Onset latency $(\mathrm{ms})$ & $2.77(0.31)$ & $2.80(0.49)$ \\
Conduction velocity $(\mathrm{m} / \mathrm{s})$ & $51.11(5.91)$ & $50.82(6.03)$ \\
\hline
\end{tabular}

Table 2 summarizes the changes in the median and ulnar NCS parameters over 5 years adjusted for hand (dominant versus nondominant), age, gender, and BMI. The changes were linearly related to duration of follow-up. The median and ulnar SNAP amplitudes decreased 1.75-2.3 $\mu \mathrm{V}$, peak latencies increased $0.06-0.11 \mathrm{~ms}$, and onset latency increased $0.043-0.072 \mathrm{~ms}$ over 5 years. The conduction velocities decreased by about $0.71-1.1 \mathrm{~m} / \mathrm{s}$ over this time.

The interaction terms between time and age were not significantly associated with parameter changes over time (all $P$-values $>0.1$ ). Thus, the subject's initial age did not significantly affect the rate of change in NCS parameters. The interaction terms between time and each of the other potential predictor variables were not associated with parameter changes over time. Thus, the changes in median and ulnar NCS parameters over time were not affected by the hand tested (dominant versus nondominant hand), gender, age, height, or BMI of the subject.
All the median and ulnar NCS parameters were significantly related to time squared ( $P$-values ranging from $<0.0001$ to 0.047 ) with the exception of the median SNAP peak latency $(P=0.18)$, the ulnar SNAP amplitude $(P=0.18)$, and the ulnar SNAP peak latency $(P=0.067)$. This suggests a nonlinear relationship between the parameters and time.

Post Hoc Analysis. After reviewing the results, it was noted that the estimated median NCS parameter changes were larger than those of the ulnar nerve. A post hoc analysis was performed to determine whether the changes were statistically different. The interaction term "time*nerve" was added to the base model to determine whether the changes over time for the median and ulnar nerves were different. The interaction term was significant for two of the four parameters: it was 0.08 for the amplitude, 0.0016 for the peak latency, 0.037 for the onset latency, and 0.077 for the conduction velocity, indicating that the rate of change of some of the parameters for the median sensory response was greater than for the ulnar sensory response.

\section{DISCUSSION}

The relationship between aging and NCS parameter changes has been studied many times, but previous studies were limited by cross-sectional study designs, which could not determine whether the changes noted were more related to aging or to age of subjects. We used data gathered from each subject at two points in time to estimate the expected change in the NCS parameters over time.

Our results show that sensory NCS parameters do change with time. Changes in NCS were primarily related linearly to the duration of follow-up. However, because both time and time-squared were sig-

\begin{tabular}{|c|c|c|c|}
\hline \multirow[b]{2}{*}{ Parameter } & \multicolumn{3}{|c|}{ Change over 5 years } \\
\hline & Change & $P$-value & $95 \% \mathrm{Cl}$ \\
\hline \multicolumn{4}{|l|}{ Median sensory NCS } \\
\hline Amplitude $(\mu \mathrm{V})$ & -2.30 & $<.0001$ & $(-3.0,-1.5)$ \\
\hline Peak latency (ms) & 0.11 & $<.0001$ & $(0.071,0.14)$ \\
\hline Onset latency (ms) & 0.072 & $<.0001$ & $(0.037,0.11)$ \\
\hline Conduction velocity $(\mathrm{m} / \mathrm{s})$ & -1.1 & $<.0001$ & $(-1.49,-0.62)$ \\
\hline \multicolumn{4}{|l|}{ Ulnar sensory NCS } \\
\hline Amplitude $(\mu \mathrm{V})$ & -1.75 & $<.0001$ & $(-2.5,-1.0)$ \\
\hline Peak latency (ms) & 0.059 & .0004 & $(0.027,0.092)$ \\
\hline Onset latency (ms) & 0.043 & .011 & $(0.010,0.076)$ \\
\hline Conduction velocity $(\mathrm{m} / \mathrm{s})$ & -0.71 & .002 & $(-1.15,-0.27)$ \\
\hline
\end{tabular}

*Other covariates in the regression model included hand used, gender, age, height, and BMI of the subject. 
nificantly related to some of the changes in NCS parameters, these may be best represented by a nonlinear relationship, as suggested by Taylor. ${ }^{21}$ Because a nonlinear relationship is hard to conceptualize or model, and the betas for the quadratic term were only one tenth the size of those for the linear term, we have presented only betas for the linear relationship. The amount of change noted was small, raising concern that some of the findings may be due to measurement error. In an earlier study, the same examiners tested 158 subjects 3 weeks apart and found good reliability, with an intraclass correlation (ICC) of 0.76-0.92 for the median sensory response parameters. ${ }^{16}$ The ulnar SNAP amplitude also had a fairly good ICC, of 0.68-0.8, but the ulnar SNAP onset and peak latencies had poor ICCs, of 0.220.43. However, even with the poor ICG with the ulnar nerve, we expect that this variability, when averaged among 440 subjects, would approach zero.

There have been three previous cross-sectional studies of the relationship between age and the median sensory conduction velocity. $5,8,19$ The study by Dorfman and Bosley ${ }^{5}$ of 30 normal subjects (15 young and 15 older adults) estimated a decrease of $0.16 \mathrm{~m} / \mathrm{s}$ per year of age, that by Stetson et al. ${ }^{19}$ of 105 normal workers (excluding workers on jobs thought to involve repetitive or forceful hand exertions) estimated a decrease of $0.13 \mathrm{~m} / \mathrm{s}$ per year, and that by Letz and Gerr $^{8}$ of over 4000 veterans estimated a decrease of $0.13 \mathrm{~m} / \mathrm{s}$ per year. We noted a slightly larger decrease in conduction velocity of 0.21 $\mathrm{m} / \mathrm{s}$ per year of follow-up. It should be noted that their studies represent change per year of age, whereas the current study demonstrates change per year of follow-up. From our baseline data, we were also able to estimate the change in velocity per year of age when all other variables were held constant. The predicted decrease in conduction velocity was $0.14 \mathrm{~m} / \mathrm{s}$ per year of age, which is similar to the conclusions from the other studies.

Only two previous cross-sectional studies examined the relationship between ulnar sensory conduction velocity and age. A decrease in conduction velocity of $0.099 \mathrm{~m} / \mathrm{s}$ per year of age ${ }^{19}$ and of $0.16 \mathrm{~m} / \mathrm{s}$ per year of age were reported. ${ }^{8}$ We estimated a decrease of $0.14 \mathrm{~m} / \mathrm{s}$ per year of follow-up. Our baseline data estimates the ulnar sensory conduction velocity to decrease by $0.13 \mathrm{~m} / \mathrm{s}$ per year of age $(95 \%$ confidence interval $[\mathrm{CI}]$ : $-0.16,-0.10)$.

The second purpose of our study was to determine whether the change in electrophysiological parameters over time was affected by the subject's initial age. Our results show that changes in the parameters were primarily related to time of fol- low-up rather than initial age of the subject. We also attempted to determine whether any other factors affected the electrophysiological parameters over time, but gender, age, BMI, and hand (dominant versus nondominant) did not do so. We had thought that the dominant hand would be exposed to more stress and would therefore exhibit greater changes over time. We had also predicted that age would have an effect because aging is associated with decreased physiological functioning including muscle functioning and regeneration., ${ }^{3,18}$ A study with a longer follow-up time might allow for detection of smaller effect sizes.

The post hoc analysis supported our impression that change occurred at a greater rate in median than in ulnar sensory nerve parameters. However, the current study cannot determine which factors caused the change. The difference may have been due to increased susceptibility of the median nerve to repetitive motion trauma or higher intracarpal canal pressures with contract stress and awkward wrist postures. ${ }^{10,20}$ These findings have not been reported previously. Only two previous cross-sectional studies evaluated both the median and ulnar sensory NCS conduction velocities in the same persons. Stetson et al. ${ }^{19}$ noted the median conduction velocity to decrease by $0.13 \mathrm{~m} / \mathrm{s}$ per year of age $(\mathrm{SD}, 0.32 ; n=$ 105) and the ulnar conduction velocity to decrease by $0.099 \mathrm{~m} / \mathrm{s}$ per year of age (SD, $0.29 ; n=105)$. Although the trend is similar to our results, an unpaired Student's $t$-test of the two values shows this is not significant $(P$-value $=0.46)$. Because Letz and Gerr $^{8}$ did not show standard deviations or standard errors of their parameter estimates, we could not infer whether changes in the median and ulnar nerves were different. The findings of the current study may differ from those of Stetson et al. ${ }^{19}$ because we followed active workers, whereas they excluded workers who performed at jobs "requiring repetitive or forceful hand exertions."

When interpreting our results, several limitations should be considered. We evaluated only sensory nerves in the arms, so our findings may not be as applicable to nerves in the legs or to motor nerves. The median sensory nerve, which is susceptible to compression at the wrist, may not represent the rate of change expected with other sensory nerves. As the follow-up period was for only 5 years, a longer follow-up period may provide a more accurate estimation of nerve conduction parameter changes over time. Finally, because only healthy subjects were evaluated, our results may not as accurately predict the rate of change in NCS parameters in subjects with diseased nerves (e.g., peripheral neuropathy) or 
metabolic-toxic disorders that may affect nerve function. ${ }^{1,8,12,22}$

\section{REFERENCES}

1. Albertazzi A, Cappelli P, Di Marco T, Maccarone M, Di Paolo B. The natural history of uremic neuropathy. Contrib Nephrol 1988;65:130-137.

2. American Association of Electrodiagnostic Medicine, American Academy of Neurology, American Academy of Physical Medicine and Rehabilitation. Practice parameter for electrodiagnostic studies in carpal tunnel syndrome: summary statement. Muscle Nerve 1993;16:1390-1391.

3. Carlson BM. Muscle regeneration and aging. Monogr Dev Biol 1992;23:189-195.

4. Denys EH. The influence of temperature in clinical neurophysiology. Muscle Nerve 1991;14:795-811.

5. Dorfman LJ, Bosley TM. Age-related changes in peripheral and central nerve conduction in man. Neurology 1979;29:3844.

6. Ganeriwal SK, Reddy BV, Surdi AD, Kowale AN, Zawar PB. Influence of age on motor nerve conduction. Indian J Physiol Pharmacol 1983;27:337-341.

7. LaFratta CW, Zalis A. Age effects on sural nerve conduction velocity. Arch Phys Med Rehabil 1973;54:475-477.

8. Letz R, Gerr F. Covariates of human peripheral nerve function. I. Nerve conduction velocity and amplitude. Neurotoxicol Teratol 1994;16:95-104.

9. Lucci RM. The effects of age on motor-nerve conduction velocity. Phys Ther 1969;49:973-976.

10. Luchetti R, Schoenhuber R, Nathan P. Correlation of segmental carpal tunnel pressures with changes in hand and wrist positions in patients with carpal tunnel syndrome and controls. J Hand Surg [Br] 1998;23:598-602.

11. Mackenzie RA, Phillips LH II. Changes in peripheral and central nerve conduction with aging. Clin Exp Neurol 1981; 18:109-116.
12. Mansouri B, Adybeig B, Rayegani M, Yasami S, Behshad V. Uremic neuropathy and the analysis of electrophysiological changes. Electromyogr Clin Neurophysiol 2001;41:107-115.

13. Rivner MH, Swift TR, Malik K. Influence of age and height on nerve conduction. Muscle Nerve 2001;24:1134-1141.

14. Rutkove SB. Effects of temperature on neuromuscular electrophysiology. Muscle Nerve 2001;24:867-882.

15. Salerno DF, Franzblau A, Werner RA, Bromberg MB, Armstrong TJ, Albers JW. Median and ulnar nerve conduction studies among workers: normative values. Muscle Nerve 1998; 21:999-1005.

16. Salerno DF, Werner RA, Albers JW, Becker MP, Armstrong TJ, Franzblau A. Reliability of nerve conduction studies among active workers. Muscle Nerve 1999;22:1372-1379.

17. SAS 8.0. Cary, North Carolina.

18. Spirduso WW. Physical dimensions of aging. U.S.A.: Human Kinetics; 1995.

19. Stetson DS, Albers JW, Silverstein BA, Wolfe RA. Effects of age, sex, and anthropometric factors on nerve conduction measures. Muscle Nerve 1992;15:1095-1104.

20. Szabo RM, Chidgey LK. Stress carpal tunnel pressures in patients with carpal tunnel syndrome and normal patients. J Hand Surg [Am] 1989;14:624-627.

21. Taylor PK. Non-linear effects of age on nerve conduction in adults. J Neurol Sci 1984;66:223-234.

22. The Diabetes Control and Complications Trial Research Group. Effect of intensive diabetes treatment on nerve conduction in the Diabetes Control and Complications Trial. Ann Neurol 1995;38:869-880.

23. Trojaborg WT, Moon A, Andersen BB, Trojaborg NS. Sural nerve conduction parameters in normal subjects related to age, gender, temperature, and height: a reappraisal. Muscle Nerve 1992;15:666-671.

24. Werner RA, Gell N, Franzblau A, Armstrong TJ. Prolonged median sensory latency as a predictor of future carpal tunnel syndrome. Muscle Nerve 2001;24:1462-1467. 